\title{
LA LEISHMANIOSE EN GRÈCE : Les Phlébotomes des Iles Ioniennes et de la mer Égée
}

\author{
B. PESSON*, N. LEGER** et G. MADULO-LEBLOND***
}

RÉSUMÉ. Trois enquêtes entomologiques ont été effectuées durant les étés 1979, 1980 et 1982 dans les îles Ioniennes de Corfou, Céphalonie et Zante et dans quatre îles de la mer Égée : à l'ouest Andros et Tinos (Cyclades) et à l'est Samos et Ikaria. Un échantillonnage systématique pratiqué par la technique des pièges adhésifs a permis la récolte de 24184 phlébotomes. Les captures sont analysées espèce par espèce.

\section{Leishmaniasis in Greece : The sandflies of Ionian and Aegean Sea Islands.}

SUMMARY. Three entomological investigations have been carried out during the summers 1979, 1980 and 1982 in Ionian islands : Corfu, Cephalonia and Zante and in four Aegean Sea islands : the western Andros and Tinos (Cyclades) and eastern Samos and Ikaria. Systematic sampling with oiled paper traps produced 24 184 sandflies. Captures are analysed for each species.

Jusqu'en 1979, mise à part l'étude du foyer leishmanien de Crète (Adler et coll., 1938), les données sur les phlébotomes de Grèce demeuraient très fragmentaires. Alors que la prévalence de la leishmaniose canine atteint un niveau critique dans certaines provinces (Artavanis et coll., 1982 ; Saratsiotis, communication personnelle) et que le nombre de cas humains augmente chaque année, il nous a paru utile de compléter l'inventaire des phlébotomes dans des régions non étudiées et présentant un intérêt épidémiologique. Dans notre première enquête effectuée en 1977, nous donnions les résultats d'une prospection entomologique en Grèce continentale, de Kozani à Athènes, et précisions la répartition des cas humains de leishmaniose dans les différents départements (Léger et coll., 1979).

Des trois foyers principaux de leishmaniose viscérale : Attique, Argolide et région Nord-Ouest, ce dernier foyer situé à l'ouest de la chaîne du Pinde et comprenant les départements de Janina, Preveza, Thesprotia et l'île de Corfou, apparaît

\footnotetext{
* Laboratoire de Parasitologie de la Faculté de Pharmacie de Strasbourg, F 67400 Strasbourg.

** Laboratoire de Parasitologie de la Faculté de Pharmacie de Reims, F 51096 Reims Cedex.

*** Laboratoire de Parasitologie de la Faculté de Pharmacie de Paris XI, F 92290 Chatenay-Malabry. Accepté le 8 juillet 1983 .
} 
remarquable par sa stabilité. Il pourrait s'agir d'un foyer d'endémie à partir duquel prendraient naissance des vagues épidémiques vers l'est et le sud. Ceci justifie dans notre enquête le choix de Corfou où 7 cas humains de leishmaniose viscérale ont été déclarés aux services d'hygiène entre 1975 et 1981. Parmi les autres îles Ioniennes, Zante apparaît comme le principal foyer de leishmaniose cutanée de Grèce (Stratigos et coll., 1980). A Céphalonie, au contraire, la situation est semblable à celle de Corfou.

A l'opposé, l'incidence de la leishmaniose est peu importante dans les îles de la mer Égée. Dans les Cyclades du Nord, aucun cas humain récent n'est signalé. Dans la province de Samos, Ikaria constitue un foyer de faible endémie : 2 cas de leishmaniose cutanée entre 1975 et 1979 (Stratigos et coll., 1980) et un cas de leishmaniose viscérale en 1981, (communication des services vétérinaires de Samos).

Les phlébotomes de ces deux groupes d'îles ont été étudiés de 1979 à 1982. En juillet 1979, une première enquête a permis de dresser l'inventaire des espèces à Corfou, Céphalonie et Zante. Elle fut complétée en 1980 par une exploration plus détaillée du foyer corfouan et en 1981 par une étude éthologique (Madulo-Leblond, 1983). En juillet 1982, quatre îles de la mer Égée ont été prospectées : à l'ouest Andros et Tinos (Cyclades) et à l'est Samos et Ikaria (fig. 1).

\section{I - Description des îles étudiées}

Situées entre le $37^{\mathrm{e}}$ et le $40^{\mathrm{e}}$ degré de latitude nord, les sept îles prospectées, présentent des contrastes biogéographiques :

- profil géographique : aux îles occidentales calcaires, s'opposent les massifs cristallins de la mer Égée :

- Les îles Ioniennes sont constituées de terrains crétacés et tertiaires de nature essentiellement calcaire. Corfou, la plus septentrionale présente des paysages variés : plaines cultivées et collines au sud, montagnes au nord, dominées par le Pantocrator (906 m). Son climat est doux et humide. Céphalonie lui est comparable, mais possède un relief plus accusé. Le mont Aenos y culmine à $1602 \mathrm{~m}$. Zante se différencie par une altitude moyenne inférieure et un climat sec.

- Les îles de la mer Égée représentent les fragments d'un socle primaire de roches éruptives et métamorphiques. Andros et Tinos sont essentiellement formées de massifs montagneux occupant le centre des îles et de petites plaines côtières. Comme les autres Cyclades, leur climat est doux et sec et elles sont souvent, en été, balayées par des vents violents (Meltem). Samos rappelle Corfou par son climat et ses paysages ; le relief dominé par le mont Kirkos (1 $440 \mathrm{~m}$ ) délimite trois dépressions principales d'origine tertiaire correspondant aux zones cultivées. Ikaria se caractérise par une altitude moyenne élevée, la rareté des plaines côtières et un climat plus aride.

- végétation : à la flore dense et variée de l' "Ile Verte " (Corfou) et de Samos, s'oppose la "phrygana " des Cyclades et d'Ikaria. Dans chaque ile, on retrouve, plus ou moins altérées, les strates caractéristiques de la végétation méditerranéenne : 


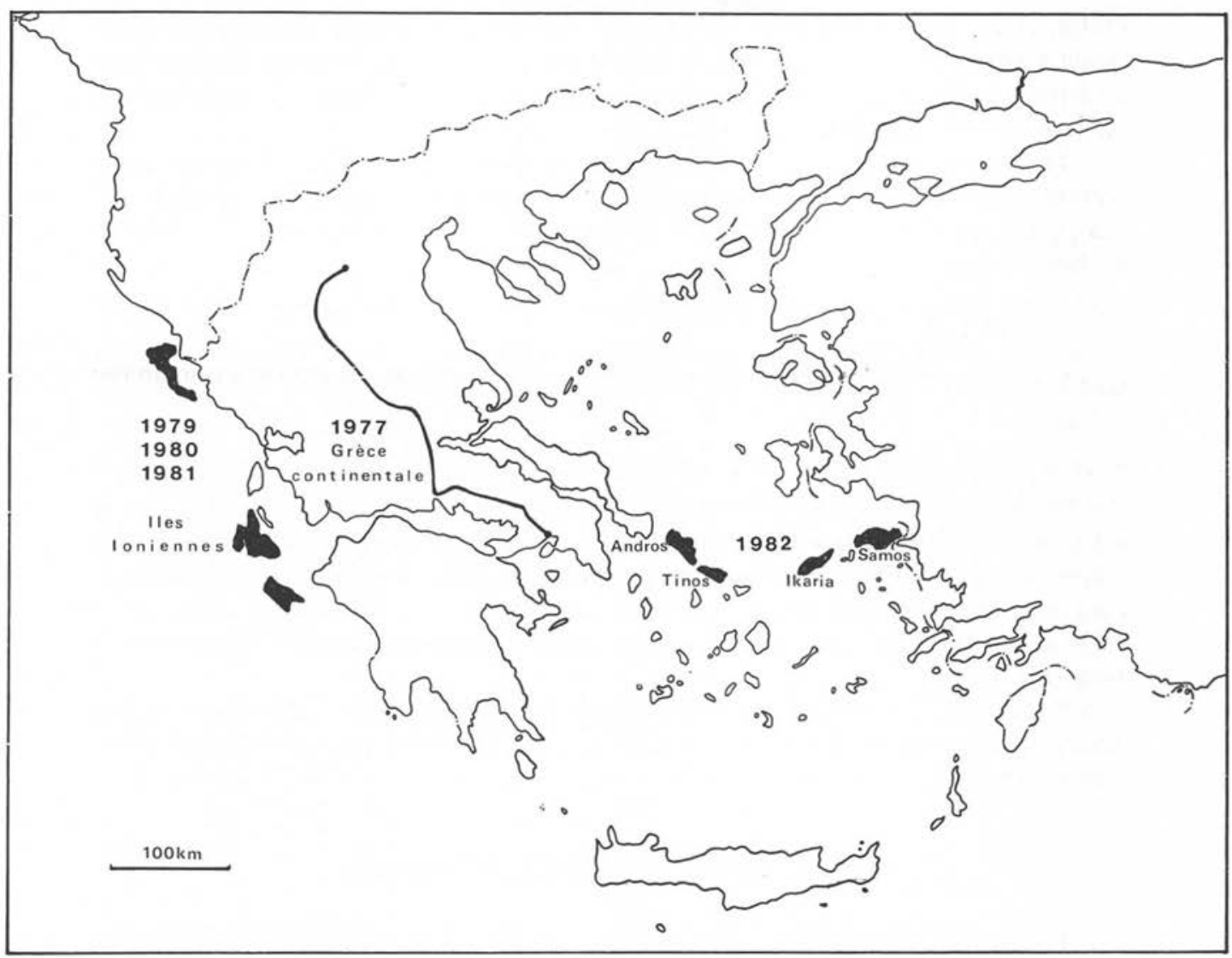

FIG. I. - Régions de Grèce prospectées de I977 à I982.

- Au-dessus de $1000 \mathrm{~m}$, altitude que nous avons prospectée uniquement à Céphalonie (sans succès d'ailleurs), c'est la forêt de Sapin de Grèce (Abies cephalonica).

- A moyenne altitude, c'est l'étage des chênes caducifoliés : Quercus macrolepis largement distribué, associé à Quercus robur à Corfou, et à Quercus pubescens à Ikaria. A Samos, au même étage, Pinus brutia est également présent.

- La strate inférieure est celle du maquis de Chêne Kermès (Quercus coccifera) associé au Cyprès (Cupressus sempervirens) dans les îles Ioniennes et au Caroubier (Ceratonia siliqua) à Samos. En terrain calcaire, c'est aussi l'étage du Pin d'Alep (Pinus halepensis).

Mais ce maquis est clairsemé et le paysage végétal le plus fréquent, à ce niveau, est celui d'une association végétale ouverte de type "garrigue ». Sur sol calcaire, (îles Ioniennes), les espèces les plus communes sont le Thym, le Romarin, le Téré- 
binthe et le Genêt d'Espagne. Sur terrain siliceux (Cyclades) : le Lentisque et le Genêt épineux. La dégradation de la végétation peut parfois aboutir à des paysages de friches herbeuses, propices à l'élevage (Tinos), ou de "lande " à peuplements paucispécifiques (Ikaria).

Traditionnellement sont cultivés la vigne (Samos) et l'olivier (Corfou), mais certaines régions présentent des cultures plus denses et diversifiées; c'est le cas des plaines de Ropa (station $n^{\circ}$ 137) à Corfou, de Hora (station no 71) à Samos et de Komi (station no 53) à Tinos.

- habitat : sa variété est liée à des facteurs économiques et ethniques.

- En zone montagneuse, il est souvent dispersé (Andros), sauf à Tinos, où la communauté catholique romaine, très importante, est regroupée dans les villages de l'intérieur.

- En région agricole, les bourgs se situent, soit au milieu des plaines cultivées (Tinos), soit au flanc des versants montagneux dominant la vallée (Samos, Corfou).

- La zone côtière est la plus peuplée : à Corfou, sont groupés typiquement sous le même nom : les villages " du haut " ("Ano ») et les petits ports " du bas " ("Kato ») dont certains sont devenus des stations touristiques très fréquentées. C'est dans cette zone côtière que se situent les villes correspondant aux principaux ports où le trafic est intense.

A Céphalonie, les villages nouveaux ont été reconstruits à une petite distance des ruines résultant du tremblement de terre de 1954, site idéal pour la pullulation des phlébotomes.

\section{II - Méthode d'échantillonnage}

La méthode de piégeage utilisée a été la capture aux pièges adhésifs (Croset et coll., 1977), particulièrement bien adaptée à l'inventaire qualitatif et quantitatif en région méditerranéenne. Les papiers, enduits d'huile de ricin, sont placés à l'entrée d'éventuels gîtes de repos domestiques et sauvages et sont relevés au bout de trois ou quatre nuits.

Dans chaque île, l'échantillonnage a été effectué sur la base de piégeages stationnels le long d'itinéraires-transects. Les stations ont été choisies de façon à explorer les diverses zones bioclimatiques et en tenant compte, dans la mesure du possible, des données recueillies sur les cas récents de leishmaniose. Au total, les transects des îles Ioniennes comprennent 148 stations, ceux des îles de la mer Égée 121. (fig. 2, 3 et 4).

FIG. 2. - Transects des Iles Ioniennes.

En noir : stations d'altitude supérieure à ${ }_{5} 50 \mathrm{~m}$. 


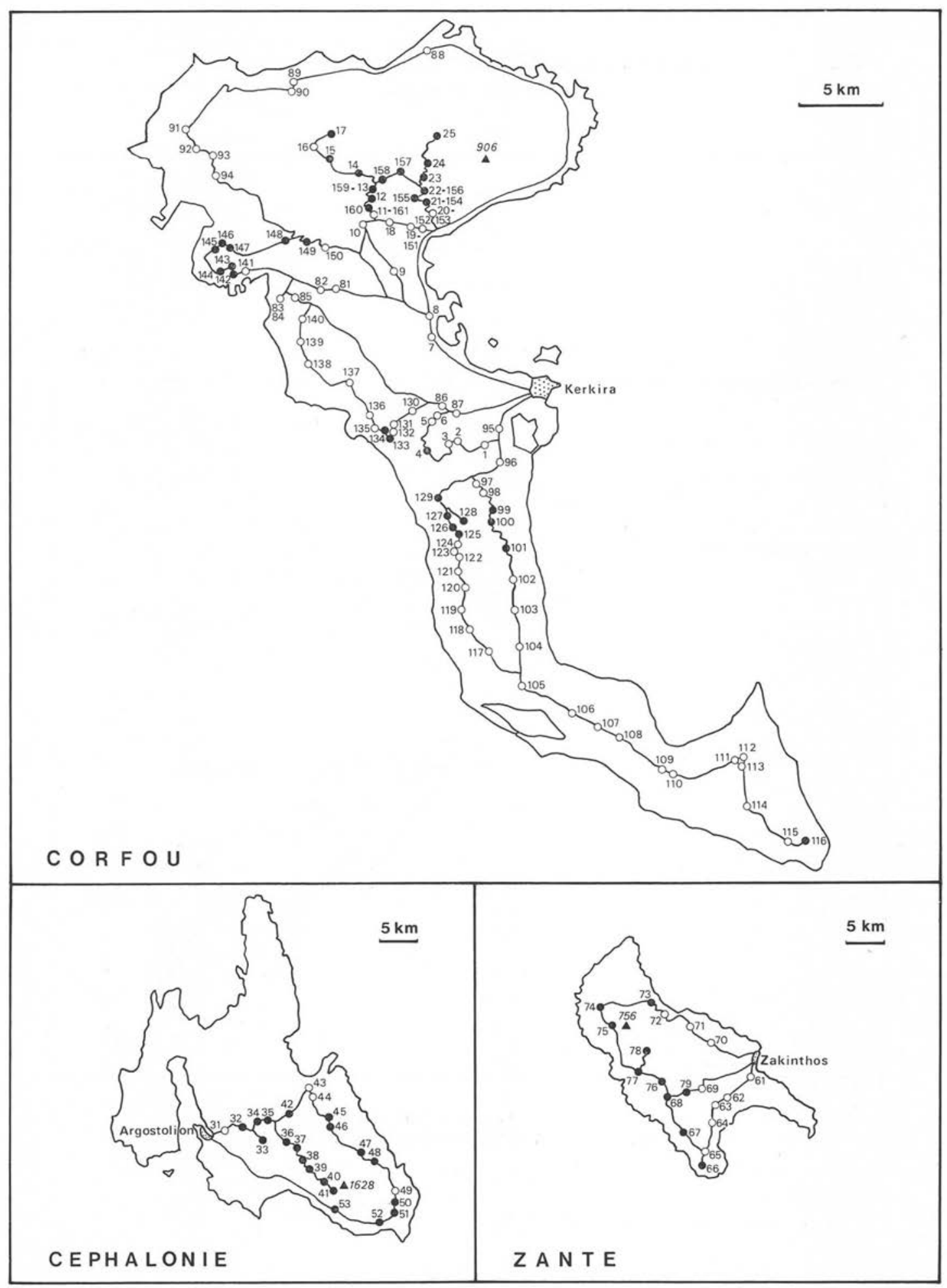




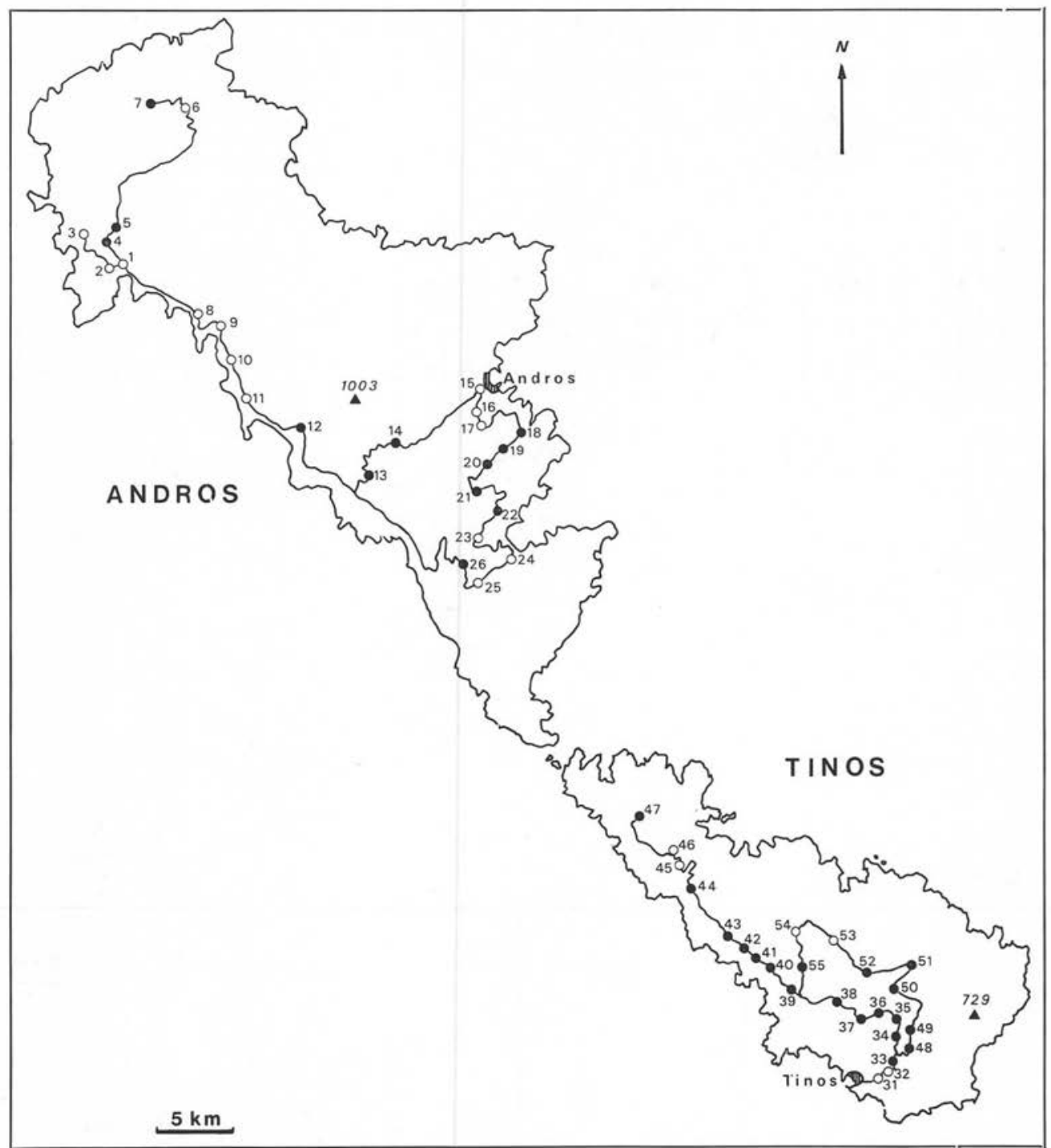

FIG. 3. - Transects des Cyclades.

En noir : stations d'altitude supérieure à $150 \mathrm{~m}$. 


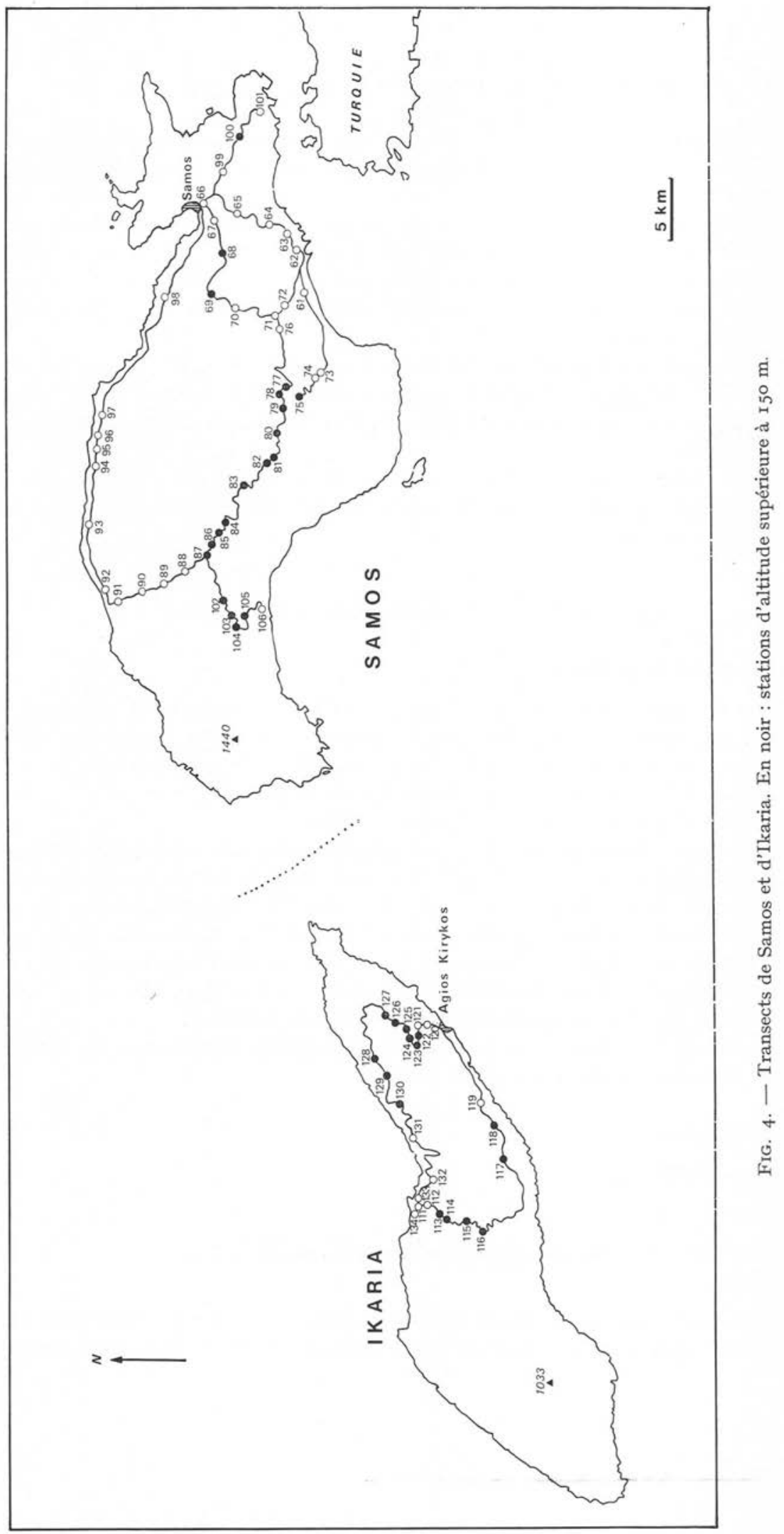


La distance moyenne entre les stations est de $3 \mathrm{~km}$. Corfou et Samos ont fait l'objet d'une investigation plus étendue que les autres îles (respectivement 106 et 46 stations).

Le nombre de pièges placés dans chaque station varie (de 2 à 116) en fonction du nombre d'anfractuosités favorables à la pose. Sur 5000 papiers placés pendant les trois enquêtes, 4739 ont été récupérés, soit 95\%. La mise en place, comme le ramassage, ont toujours eu lieu au mois de juillet. Au total 24184 phlébotomes ont été récoltés par cette méthode.

Les exemplaires capturés ont été recueillis et conservés dans l'alcool. Les identifications ont été faites sur montages permanents dans le baume, après éclaircissement à la potasse à $20 \%$ et au liquide de Marc-André, suivi d'une coloration à la fuchsine acide.

Dans plusieurs stations, des captures manuelles et au piège lumineux ont été pratiquées. Elles nous ont apporté des renseignements complèmentaires sur l'éthologie de certaines espèces.

\section{III - Analyse des captures}

\section{1 - Inventaire faunistique}

Des 10 espèces de phlébotomes décrites en Grèce continentale (Léger et coll., 1979), 8 seulement sont récoltées dans les deux groupes d'îles prospectées. Elles se répartissent en deux genres et cinq sous-genres. N'ont pas été retrouvés :

Phlebotomus (Paraphlebotomus) alexandri Sinton, 1928 et

Phlebotomus (Adlerius) balcanicus Theodor, 1958.

Le tableau $I$ donne les résultats des captures espèce par espèce, dans chaque île.

Les tableaux II et III montrent l'abondance relative de chaque espèce dans les deux groupes d'îles en faisant une distinction entre les Sergentomyia, dont les préférences trophiques limitent leur rôle éventuel dans la transmission des leishmanioses et les Phlebotomus. Dans ce dernier genre, la distinction est également faite entre $P$. sergenti, un des vecteurs de leishmaniose cutanée, et le groupe Larroussius-Adlerius qui renferme des vecteurs potentiels de leishmaniose viscérale.

Le tableau IV résume, pour chaque île, les proportions respectives des différentes espèces, à l'intérieur du genre Phlebotomus.

\section{2 - Commentaires}

\section{A - Genre Phlebotomus}

a - Sous-genre Phlebotomus

- Phlebotomus (Phlebotomus) papatasi (Scopoli, 1786)

- Chorologie

Deux exemplaires seulement, ont été capturés dans les îles Ioniennes : un à Céphalonie (station no 34) et un à Corfou (station no 128). Comme en Grèce conti- 


\begin{tabular}{|c|c|c|c|c|c|c|c|c|c|}
\hline 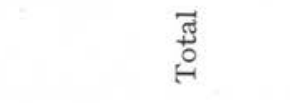 & & $\begin{array}{l}\stackrel{\infty}{\sim} \\
\stackrel{+}{\simeq}\end{array}$ & $\begin{array}{l}\text { Ðै } \\
\text { ข }\end{array}$ & $\begin{array}{l}0 \\
⿱ \\
0\end{array}$ & ֻ & & ֻ & రి & $\begin{array}{l}\stackrel{4}{ \pm} \\
\stackrel{\sim}{+}\end{array}$ \\
\hline \multirow{2}{*}{$\begin{array}{l}\text { Sergentomyia } \\
\text { minuta }\end{array}$} & Ot & $\begin{array}{l}\text { 인 } \\
=\end{array}$ & ஜூ & I & ळి & & అै & กี & \multirow{2}{*}{$\begin{array}{l}\text { th } \\
\text { เू } \\
\infty\end{array}$} \\
\hline & ro & $\begin{array}{l}\stackrel{O}{0} \\
\text { iv }\end{array}$ & $\stackrel{\varphi}{\stackrel{2}{*}}$ & 尺ి & ్ㅏ & in & $\tilde{\vartheta}$ & ๓ี & \\
\hline \multirow{2}{*}{$\begin{array}{l}\text { Sergentomyia } \\
\text { dentata }\end{array}$} & ot & $\begin{array}{l}\qquad \\
\varnothing \\
\forall\end{array}$ & $\stackrel{\Re}{\beth}$ & \& & 1 & 1 & 유 & $\mathscr{\varrho}$ & \multirow{2}{*}{ 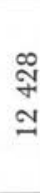 } \\
\hline & ro & $\begin{array}{l}\text { लै } \\
\text { ले } \\
\text { v }\end{array}$ & $\stackrel{\infty}{\sim}$ & $\begin{array}{l}\text { In } \\
\text { ก }\end{array}$ & 1 & 1 & ః్ & $\overrightarrow{\mathrm{N}}$ & \\
\hline \multirow{2}{*}{$\begin{array}{l}\text { Phlebotomus } \\
\text { simici }\end{array}$} & & $\stackrel{\Delta}{\sim}$ & 1 & 1 & 一 & N & $N$ & N & \multirow{2}{*}{ ర్ల్ } \\
\hline & ro & & & 1 & $\mathscr{7}$ & के & 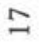 & 오 & \\
\hline \multirow{2}{*}{$\begin{array}{l}\text { Phlebotomus } \\
\text { tobbi }\end{array}$} & ot & 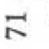 & $\omega$ & N & N & I & 一 & 1 & \multirow{2}{*}{ 茋 } \\
\hline & & ఫ్ల & คิ & $\dddot{0}$ & 0 & 1 & $\cong$ & - & \\
\hline \multirow{2}{*}{$\begin{array}{c}\text { Phlebotomus } \\
\text { perfiliewi }\end{array}$} & of & $\stackrel{\mathscr{I}}{\simeq}$ & N & $m$ & 1 & 1 & 1 & 1 & \multirow{2}{*}{ ปั } \\
\hline & ro & $\sqrt{4}$ & & $\Xi$ & 1 & 1 & - & 1 & \\
\hline \multirow{2}{*}{$\begin{array}{l}\text { Phlebotomus } \\
\text { major }\end{array}$} & of & & 음 & + & 6 & $n$ & 으 & - & \multirow{2}{*}{ छ } \\
\hline & & ণ্م & $\vec{n}$ & $\vec{m}$ & ㅇํ & สิ & $\mathscr{f}$ & คิ & \\
\hline \multirow{2}{*}{$\begin{array}{c}\text { Phlebotomus } \\
\text { sergenti }\end{array}$} & ot & \pm & $\cong$ & ลิ & $\stackrel{\sim}{\sim}$ & r & $\overline{\text { เ }}$ & $\omega$ & \multirow{2}{*}{ 令 } \\
\hline & & 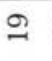 & $\stackrel{m}{F}$ & 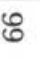 & ㅇ & 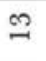 & I & $N$ & \\
\hline \multirow{2}{*}{$\begin{array}{c}\text { Phlebotomus } \\
\text { papatasi }\end{array}$} & ot & 1 & 1 & 1 & 1 & 1 & 1 & 1 & \multirow{2}{*}{ N } \\
\hline & ro & - & - & I & & 1 & 1 & I & \\
\hline Nombre de pièges & & $\begin{array}{l}\overrightarrow{\text { Iี }} \\
\text { v }\end{array}$ & ల్లి & \&્ণ & స్ & ๕̊ & ஜి & $\stackrel{\infty}{\infty}$ & $\begin{array}{l}\text { ले } \\
\text { † } \\
\text { + }\end{array}$ \\
\hline Nombre de stations & & ๕ & ขึ & 2 & $\stackrel{\sim}{\sim}$ & $\stackrel{2}{\sim}$ & $\mathscr{q}$ & $\dot{\Delta}$ & \&્ণ \\
\hline 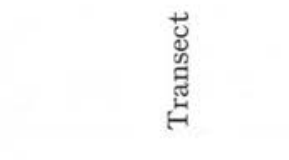 & & $\underset{\mathscr{c}}{\delta}$ & 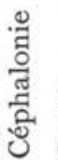 & 范 & 总 & $\stackrel{\infty}{\stackrel{0}{\Xi}}$ & 总 & 莺 & ॠే \\
\hline
\end{tabular}


TABleau II. - Abondance relative des différentes espèces dans les îles Ioniennes.

\begin{tabular}{|c|c|c|c|c|c|}
\hline Genres & Sous-Genres & Espèces & Corfou & Céphalonie & Zante \\
\hline \multirow[t]{6}{*}{ Phlebotomus } & Phlebotomus & papatasi & ( 1 exemplaire) & (1 exemplaire) & - \\
\hline & Paraphlebotomus & sergenti & $0,3 \%$ & $2,25 \%$ & $1,4 \%$ \\
\hline & Larroussius & major & $6,7 \%$ & $2,3 \%$ & $0,5 \%$ \\
\hline & & perfiliewi & $4,8 \%$ & $0,45 \%$ & $0,3 \%$ \\
\hline & & tobbi & $3,6 \%$ & $1 \%$ & $1 \%$ \\
\hline & Adlerius & simici & $1,6 \%$ & - & - \\
\hline \multirow[t]{2}{*}{ Sergentomyia } & Sergentomyia & dentata & $57,7 \%$ & $17,3 \%$ & $51,3 \%$ \\
\hline & & minuta & $25,3 \%$ & $76,7 \%$ & $45,3 \%$ \\
\hline
\end{tabular}

TABleau III. - Abondance relative des différentes espèces dans les îles de la mer Égée.

\begin{tabular}{|c|c|c|c|c|c|c|}
\hline Genres & Sous-Genres & Espèces & Andros & Tinos & Samos & Ikaria \\
\hline \multirow[t]{5}{*}{ Phlebotomus } & Paraphlebotomus & sergenti & $48,1 \%$ & $21,3 \%$ & $17,6 \%$ & 2 \\
\hline & Larroussius & major & $9,8 \%$ & $28,7 \%$ & $4,3 \%$ & $4 \%$ \\
\hline & & perfiliewi & - & - & $0,1 \%$ & - \\
\hline & & tobbi & $3 \%$ & - & $0,9 \%$ & $0,2 \%$ \\
\hline & Adlerius & simici & $16,5 \%$ & $38,3 \%$ & $1,5 \%$ & $11 \%$ \\
\hline \multirow[t]{2}{*}{ Sergentomyia } & Sergentomyia & dentata & - & - & $66 \%$ & $68,8 \%$ \\
\hline & & minuta & $22,6 \%$ & $11,7 \%$ & $9,6 \%$ & $14 \%$ \\
\hline
\end{tabular}

TABleau IV. - Répartition des espèces du genre Phlebotomus.

\begin{tabular}{|c|c|c|c|c|c|c|c|}
\hline $\begin{array}{l}\text { Nombre et } \% \text { de } \\
\text { Phlebotomus dans } \\
\text { chaque transect }\end{array}$ & $\begin{array}{c}\text { Corfou } \\
2117 \\
(16,9 \%)\end{array}$ & $\begin{array}{c}\text { Céphalonie } \\
160 \\
(6 \%)\end{array}$ & $\begin{array}{c}\text { Zante } \\
213 \\
(3,1 \%)\end{array}$ & $\begin{array}{c}\text { Andros } \\
206 \\
(77,5 \%)\end{array}$ & $\begin{array}{c}\text { Tinos } \\
83 \\
(88 \%)\end{array}$ & $\begin{array}{c}\text { Samos } \\
319 \\
(24,4 \%)\end{array}$ & $\begin{array}{c}\text { Ikaria } \\
104 \\
(17,3 \%)\end{array}$ \\
\hline P. papatasi & $0,04 \%$ & $0,6 \%$ & - & - & - & - & \\
\hline$P$. sergenti & $1,5 \%$ & $37,5 \%$ & $43,7 \%$ & $62,1 \%$ & $24,1 \%$ & $72,1 \%$ & $12,5 \%$ \\
\hline P. major & $39,6 \%$ & $38,1 \quad \%$ & $16,4 \%$ & $12,6 \%$ & $32,5 \%$ & $17,5 \%$ & $23,1 \%$ \\
\hline P. perfiliewi & $28,1 \%$ & $7,5 \%$ & $8 \%$ & - & - & $0,3 \%$ & - \\
\hline P. tobbi & $21,5 \%$ & $16,3 \%$ & $31,9 \%$ & $3,9 \%$ & - & $4,1 \%$ & $0,9 \%$ \\
\hline P. simici & $9,3 \%$ & - & - & $21,4 \%$ & $43,4 \%$ & $6 \%$ & $63,5 \%$ \\
\hline
\end{tabular}


nentale (Léger et coll., 1979), on est frappé par le petit nombre de captures d'une espèce anthropophile et endophile, pourtant largement répandue sur le pourtour méditerranéen et signalée à Corfou même, par Stephanides (1939).

A cela, plusieurs explications peuvent être données : un trop petit nombre de gîtes domestiques explorés, une sortie plus tardive dans la saison ou une rareté réelle pouvant être due à la lutte insecticide domiciliaire, généralisée en Grèce, même en dehors des zones touristiques. Cette dernière hypothèse nous semble la plus probable : au cours d'une étude éthologique, prolongée dans la saison, Madulo-Leblond (1983), a observé le même phénomène dans les villages de Corfou. A Athènes, où $P$. papatasi était autrefois très répandu (Hertig, 1949; Hadjinicolaou, 1958), il ne subsiste plus que quelques gîtes abrités, non soumis à l'action humaine (Saratsiosis, communication personnelle).

b - Sous-genre Paraphlebotomus

- Phlebotomus (Paraphlebotomus) sergenti Parrot, 1917.

- Chorologie

Cette espèce, présente dans les sept îles, joue probablement un rôle important dans la transmission de la leishmaniose cutanée en Grèce. Ainsi, c'est la plus abondante des espèces du genre Phlebotomus capturées à Zante, principal foyer de leishmaniose cutanée. Elle représente également la plus forte proportion des Phlebotomus récoltés à Andros et Samos où pourtant l'incidence du Bouton d'Orient est faible (Stratigos et coll., 1980).

Sa répartition, comme celle de la maladie, est sporadique : à Zante, 98\% des captures ont eu lieu dans seulement trois stations $(67,77$ et 79$)$ distantes l'une de l'autre de moins de $10 \mathrm{~km}$ et centrées par les villages d'Agios Nicolaos et de Romiri, foyers connus de leishmaniose cutanée. A Andros, on relève $80 \%$ des captures dans une seule station (station no 3 ) et à Samos $92 \%$ dans le seul village de Vathi (station $n^{0} 66$ ) à la sortie de la ville de Samos.

c - Sous-genre Larroussius

D'après ce que l'on sait de la situation dans d'autres régions méditerranéennes, trois espèces sont, dans la zone étudiée, des vecteurs potentiels de Leishmania infantum : Phlebotomus major, Phlebotomus perfiliewi et Phlebotomus tobbi.

- Phlebotomus (Larroussius) major Annandale, 1910.

- Systématique

Le binôme $P$. major recouvre un ensemble d'au moins 5 sous-espèces (Lewis, 1982). Nous avions, en 1979, identifié nos captures de Grèce continentale comme P. major syriacus (Adler et Theodor, 1931); or les îles Ioniennes sont proches des localités types de P. major neglectus Tonnoir, 1921 (Albanie et Yougoslavie). P. neglectus, mis en synonymie avec $P$. major s. str. dès 1930, par Nitzulescu n'est pratiquement jamais plus mentionné dans les relevés entomologiques de cette région du bassin méditerranéen. La plupart des auteurs utilisent l'appellation binôminale, sans 
mention de sous-espèce (Caminopetros 1934, 1935; Parrot, 1935; Hertig, 1949; Simic et Zivkovic, 1956 ; Hadjinicolaou, 1958).

En l'absence d'exemplaires de référence accessibles, nous avons pris en compte les critères morphologiques, donnés par Lewis dans sa clé d'identification (1982). Les phlébotomes grecs, quelle que soit leur origine (Corfou, Andros, Thessalie), ne présentent pas de différence entre eux, ni avec des exemplaires en provenance de Serbie et d'Italie, conservés dans nos collections. Certains caractères les rapprochent de la sous-espèce neglectus, d'autres de la sous-espèce syriacus :

- la formule des palpes des mâles et des femelles est 1-4-2-3-5.

- chez les femelles, l'armature pharyngienne occupe 40 à $65 \%$ de la longueur totale du pharynx ; la taille moyenne des ailes est de $2,7 \mathrm{~mm}$.

- chez les mâles, dont l'aedeage $(200-240 \mu \mathrm{m})$ présente la forme caractéristique en baguette de tambour, le style $(170-200 \mu \mathrm{m})$ est moitié moins long que le coxite (340-400 $\mu \mathrm{m})$. Ce dernier est toujours garni d'une trentaine de soies serrées.

Devant ces observations, il nous est impossible de préciser la sous-espèce de $P$. major récoltée. Il semble que l'existence des différentes sous-espèces de l'est méditerranéen est discutable. Il en est de même pour $P$. major krimensis : l'examen d'un exemplaire mâle de Crimée provenant de la collection de Parrot (F 416) ne révèle, au niveau du génitalia, aucune différence significative avec les autres exemplaires.

- Chorologie et biologie

$P$. major représente une proportion importante des Phlebotomus récoltés à Corfou $(39,6 \%)$, Céphalonie $(38,1 \%)$ et Tinos $(32,5 \%)$. Dans ces deux dernières îles, l'importance relative de $P$. major dans les piégeages aux papiers huilés est certainement liée au tracé des itinéraires-transects. Ceux-ci comportent des stations en majorité situées entre 200 et $600 \mathrm{~m}$ d'altitude : 17 sur 23 à Céphalonie ; 16 sur 25 à Tinos. Or c'est à cet étage qu'on note, habituellement, la plus forte densité de P. major. Cette répartition correspond également à la localisation des foyers de leishmaniose viscérale dans certaines zones d'endémie du bassin méditerranéen (Léger et coll., 1979).

A Corfou, $P$. major est largement distribué. On le capture constamment autour des habitations et des poulaillers. Il se nourrit d'ailleurs aussi bien sur oiseaux que sur mammifères, comme nous avons pu le constater en étudiant les contenus stomacaux. Des formes promastigotes, dont l'identification n'a malheureusement pas pu être menée à bien, ont même été trouvées chez une femelle capturée à Lakones (Corfou, station no 142) localité où a été signalée en 1981 un cas de leishmaniose viscérale (Madulo-Leblond, 1983). P. major, qui est un bon vecteur de leishmaniose, doit probablement, jouer un rôle important dans la transmission de la maladie en Grèce.

- Phlebotomus (Larroussius) perfiliewi Parrot, 1930.

- Systématique

Cette espèce comporte actuellement 3 sous-espèces (Perfiliev, 1968) :

P. perfiliewi perfiliewi Parrot, 1930.

P. perfiliewi transcaucasius Perfiliev, 1937.

P. perfiliewi galilaeus Theodor, 1958. 
Lewis (1982) rattache à la première sous-espèce toutes les captures rangées, par divers auteurs, sous la dénomination binominale, ce qui lui confère une répartition géographique très large, les autres sous-espèces ne peuplant au contraire que des zones limitées : Chypre, Israël et Turquie pour $P$. p. galilaeus; U.R.S.S., Iran et Irak pour $P$. p. transcaucasicus.

La diagnose de ces sous-espèces repose essentiellement sur l'étude des valves péniennes. Celles de nos exemplaires présentent une taille moyenne de $135 \mu \mathrm{m}$ comparable aux $P$. perfiliewi de Grèce continentale, de Yougoslavie et d'Italie (fig. 5). L'aspect de l'extrémité transparente, quoique variable selon les échantillons et leur orientation au montage, est souvent proche de la description donnée par Perfiliev (1968) pour P. p. galilaeus : apex étroit, allongé et incurvé. La bordure chitinisée porte 4 à 5 petites dents groupées à la base de l'apex ( $f$ ig. $5 \mathrm{~A} \mathrm{et} \mathrm{B}$ ). Cependant, la taille des valves est toujours inférieure à $150 \mu \mathrm{m}(170-190 \mu \mathrm{m}$ pour P.p. galilaeus) et, par ailleurs, la formule des ascoïdes correspond à celle de $P$. p. perfiliewi : 2/3-15. La validité de ces sous-espèces ayant déjà été mise en doute par Théodor (1958) il semble qu'un nouvel examen des exemplaires appartenant aux diverses collections s'impose comme pour $P$. major. La chimiotaxonomie permet, à l'heure actuelle une nouvelle approche de ce genre de problème. Les travaux entrepris par Ward et coll. (1981) sur les populations italiennes de $P$. perfiliewi sont prometteurs et, ainsi que le soulignent les auteurs, mériteraient d'être étendus à toute l'aire de répartition de l'espèce.

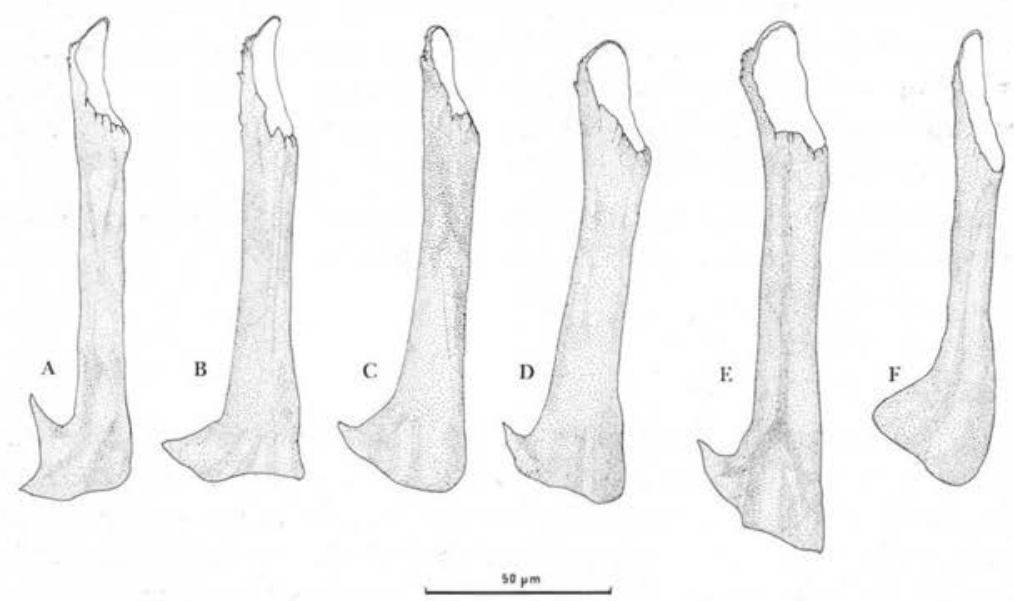

FIG. 5. - Valves péniennes de $P$. perfiliewi $s$. $l$. d'Europe.

A : Corfou, B : Samos, C : Thessalie, D : Macédoine, E : Serbie, F : Italie.

- Chorologie et biologie

P. perfiliewi, déjà signalé en Grèce continentale (Hertig, 1949 ; Léger et coll., 1979 ) est abondant dans les îles Ioniennes (28,1\% des Phlebotomus à Corfou). Au 
contraire, en mer Égée, un seul exemplaire a été capturé dans l'île de Samos, et sa présence est également exceptionnelle en Turquie (Houin et coll., 1971).

A Corfou, l'anthropophilie de $P$. perfiliewi a pu être établie par des captures sur appât humain. Bien que quelques exemplaires aient été capturés dans les poulaillers, en particulier des femelles gorgées renfermant des hématies nuclées, il s'agit ici d'une espèce peu ornithophile (beaucoup moins que $P$. major).

- Phlebotomus (Larroussius) tobbi Adler et Theodor, 1930.

- Chorologie et biologie

Présente dans toutes les îles prospectées à l'exception de Tinos, cette espèce est abondante dans les îles Ioniennes : $31,9 \%$ des Phlebotomus à Zante, $21,5 \%$ à Corfou. Elle est peu représentée dans les îles de la mer Égée, où les pourcentages des captures avoisinent ceux de Grèce continentale.

A Corfou, plusieurs femelles ont été capturées gorgées sur appât humain. P. tobbi, pourrait donc jouer dans ce foyer un rôle actif dans la transmission de la leishmaniose viscérale, comme dans d'autres régions de l'est méditerranéen et en Transcaucasie (Theodor, 1964).

$\mathrm{d}$ - Sous-genre Adlerius

- Phlebotomus (Adlerius) simici Nitzulescu, 1931.

- Systématique

Malgré la récente mise au point d'Artemiev (1980), la systématique des Adlerius comporte encore bien des incertitudes. D'abord considéré comme une sous-espèce de $P$. major (Newstead, 1916), P. chinensis, élevé au rang d'espèce par Sinton en 1928, fut assez rapidement reconnu comme étant hétérogène et divisé en sous-espèces, qu'en 1980 Artemiev transforme en autant d'espèces.

En ce qui concerne l'Europe et le Proche-Orient - si l'on excepte P. kyreniae, très localisé - trois espèces se partagent le territoire :

- P. simici Nitzulescu, 1931.

- P. balcanicus Theodor, 1958.

- P. longiductus Parrot, 1928.

Elles se différencient essentiellement : par le nombre de soies présentes sur le coxite du mâle, en moyenne 106 pour balcanicus, 64 pour longiductus, 20 pour simici (Artemiev, 1980) et par la formule des ascoïdes au niveau des antennes. Utilisant ces critères, nous avons pu vérifier l'homogénéité de nos captures d'Adlerius : ascoïdes $=2 / 3-15$, soies sur le coxite $=19$ à 35 (nombres supérieurs à ceux donnés par Artemiev sur deux exemplaires en provenance de Serbie : 19 à 22, et que nous retrouvons dans nos collections de Serbie et de Macédoine). Il s'agit donc de $P$. simici; $P$. balcanicus, signalé en Grèce continentale (Léger et coll., 1979) n'a pas été retrouvé.

- Chorologie et biologie

$P$. simici dont la limite ouest de l'aire de répartition se situe en Dalmatie près du $17^{\circ}$ de longitude, est une espèce typiquement est-méditerranéenne. Elle est peu 
abondante dans les îles Ioniennes (9,3\% des Phlebotomus à Corfou) mais occupe une place prépondérante dans les îles de la mer Égée. (63,5\% à Ikaria).

Pour dresser la carte de répartition des 3 espèces d'Europe, en l'absence d'un réexamen des collections, il convient d'éliminer un certain nombre de localisations retenues par Artemiev (1980) et, à sa suite, par Lewis (1982). Ces localisations sont sujettes à caution du fait des sources citées :

- pour $P$. balcanicus : la Crète rapportée par Hadjinicolaou (1958), mais sous le nom de $P$. chinensis

- pour $P$. simici : le Caucase et l'Asie centrale cités sans référence par Perfiliew (1968) et l'Iran rapporté par Theodor (1958) qui n'en parle plus dans son article sur les phlébotomes d'Iran en 1964.

Ces précisions données, la carte (fig. 6) fait apparaître la juxtaposition des aires de distribution, qui sont : périméditerranéenne pour $P$. simici, balcanique et caucasique pour $P$. balcanicus, septentrionale pour $P$. longiductus. Cette dernière espèce est essentiellement asiatique et "émet " en Europe un fin prolongement qui longe la Ciscaucasie et se termine en Transylvanie.

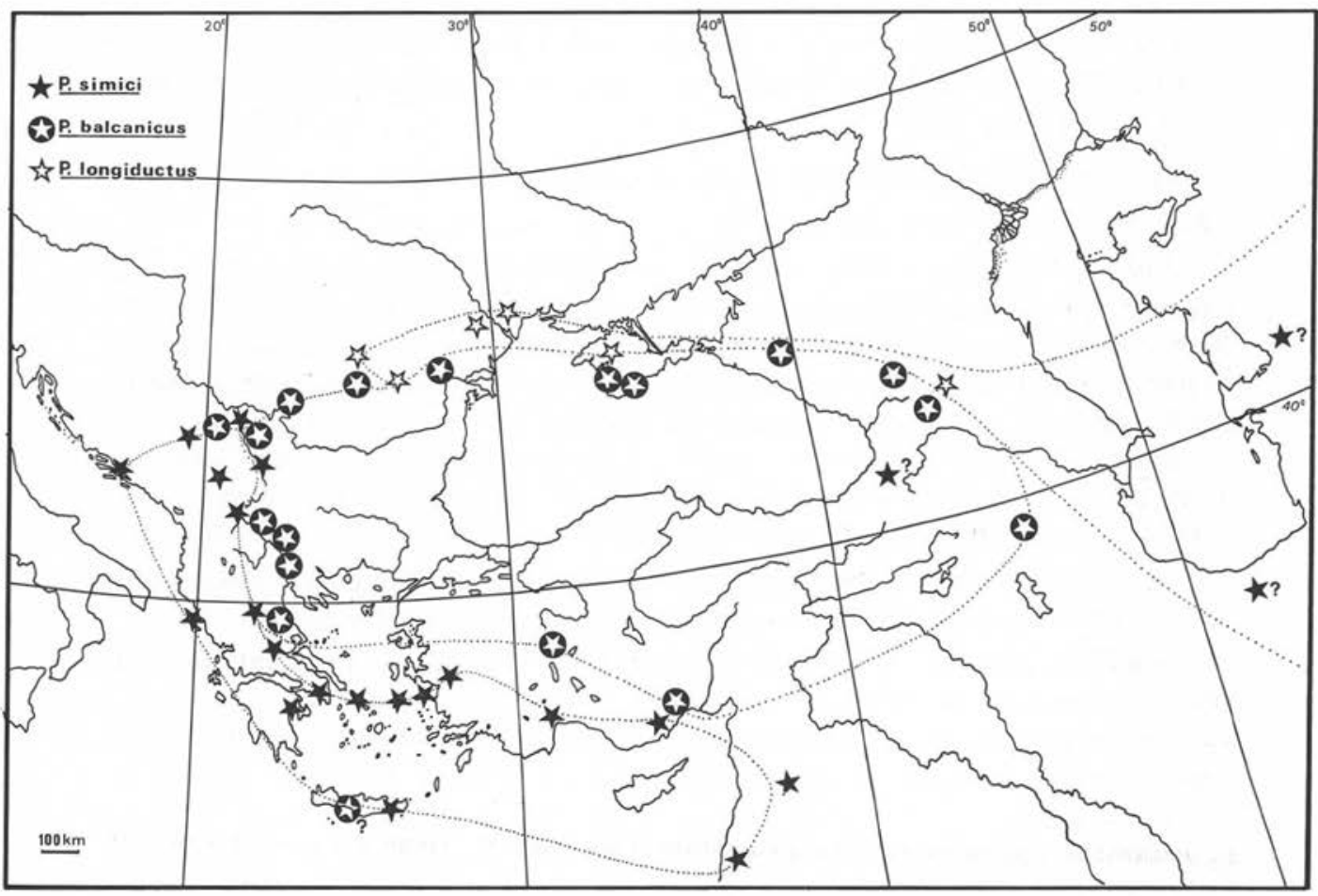

FIG. 6. - Répartition des Adlerius dans l'est-méditerranéen et le Moyen-Orient. (?: localisations sujettes à caution).

P. simici: Alpes dinariques, Pinde, Taurus.

$P$. balcanicus: Balkans, Carpates, Caucase, Taurus.

$P$. longiductus : bordure septentrionale du Caucase et de ses prolongements en Crimée-Roumanie. 
$P$. simici est peu anthropophile dans les îles prospectées. Cependant, son abondance, par rapport aux Larroussius en mer Égée (Ikaria), pourrait appeler de commentaires épidémiologiques, du fait qu'il a déjà été reconnu comme vecteurdes leishmaniose viscérale, dans les régions voisines (Theodor, 1964).

\section{B - Genre Sergentomyia}

b - Sous-genre Sergentomyia

Les Sergentomyia représentent $86,8 \%$ des captures, soit : Sergentomyia dentata $51,4 \%$ et Sergentomyia minuta $35,4 \%$.

- Sergentomyia (Sergentomyia) dentata (Sinton, 1933)

- Systématique et chorologie

Nous avons déjà expliqué l'emploi de ce binôme pour désigner les phlébotomes du groupe capturés en Grèce continentale (Léger et coll., 1979). En première analyse, les populations des îles ne présentent pas de particularité morphologique.

On observe une faible proportion de Sergentomyia dans les captures des Cyclades (tableau III) et $S$. dentata, qui est l'espèce récoltée en plus grand nombre au cours de cette enquête (tableau I), n'a pas été retrouvée à Andros et Tinos. A noter qu'elle est également peu abondante en Attique, région continentale la plus proche de ces îles (Léger et coll., 1979).

- Sergentomyia (Sergentomyia) minuta (Rondani, 1843)

- Systématique et chorologie

Chez cette espèce méditerranéenne, on distinguait classiquement deux sousespèces : S. m. minuta en Europe et S. $m$. parroti en Afrique du Nord. La distinction était fondée sur le nombre de dents présentées par l'armature cibariale des femelles : en moyenne 43 pour $S$. m. minuta et 69 pour $S$. m. parroti. Dans une étude statistique sur des captures de France, d'Espagne, du Maroc et de Tunisie, Rioux et coll. (1975) ont montré que cette variation du nombre de dents cibariales correspond à un cline nord-sud en rapport avec un gradient climatique. Récemment, Belazzoug et coll., (1982), précisent que l'augmentation numérique de ces dents est liée à l'aridification.

S. minuta est présent dans toutes les îles prospectées. En étudiant nos captures de Grèce continentale (Kalambaka) et insulaire, nous constatons que :

- l'armature cibariale des femelles présente un nombre de dents intermédiaire entre les deux sous-espèces.

- ce nombre croît du nord au sud, avec des résultats identiques dans les régions situées à la même latitude et au climat comparable (fig. 7).

La carte (fig. 8) donne les valeurs moyennes du nombre de dents cibariales de $S$. minuta, relevées autour de la Méditerranée (Rioux et coll., 1975 ; Léger et coll., 1979 ; Belazzoug et coll., 1982). Les résultats de Grèce viennent confirmer l'existence du cline nord-sud de $S$. minuta, démontrée dans la partie occidentale du bassin méditerranéen. 

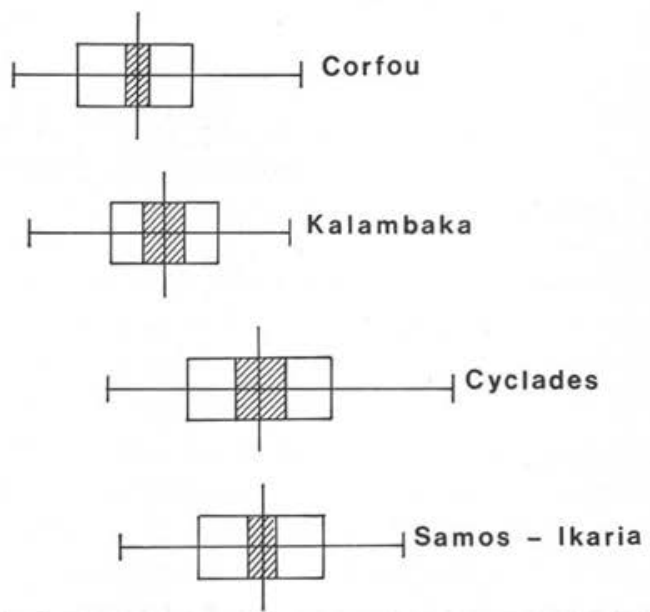

FIG. 7. - Dents cibariales de S. minuta en Grèce ; trait vertical : moyenne de l'échantillon - rectangle hachuré: intervalle de confiance $(95 \%)$ de la moyenne - rectangle blanc : écarttype ligne horizontale : extrêmes

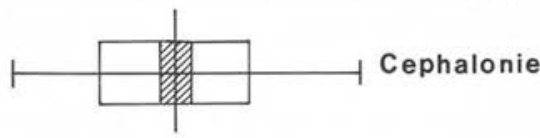
observés.
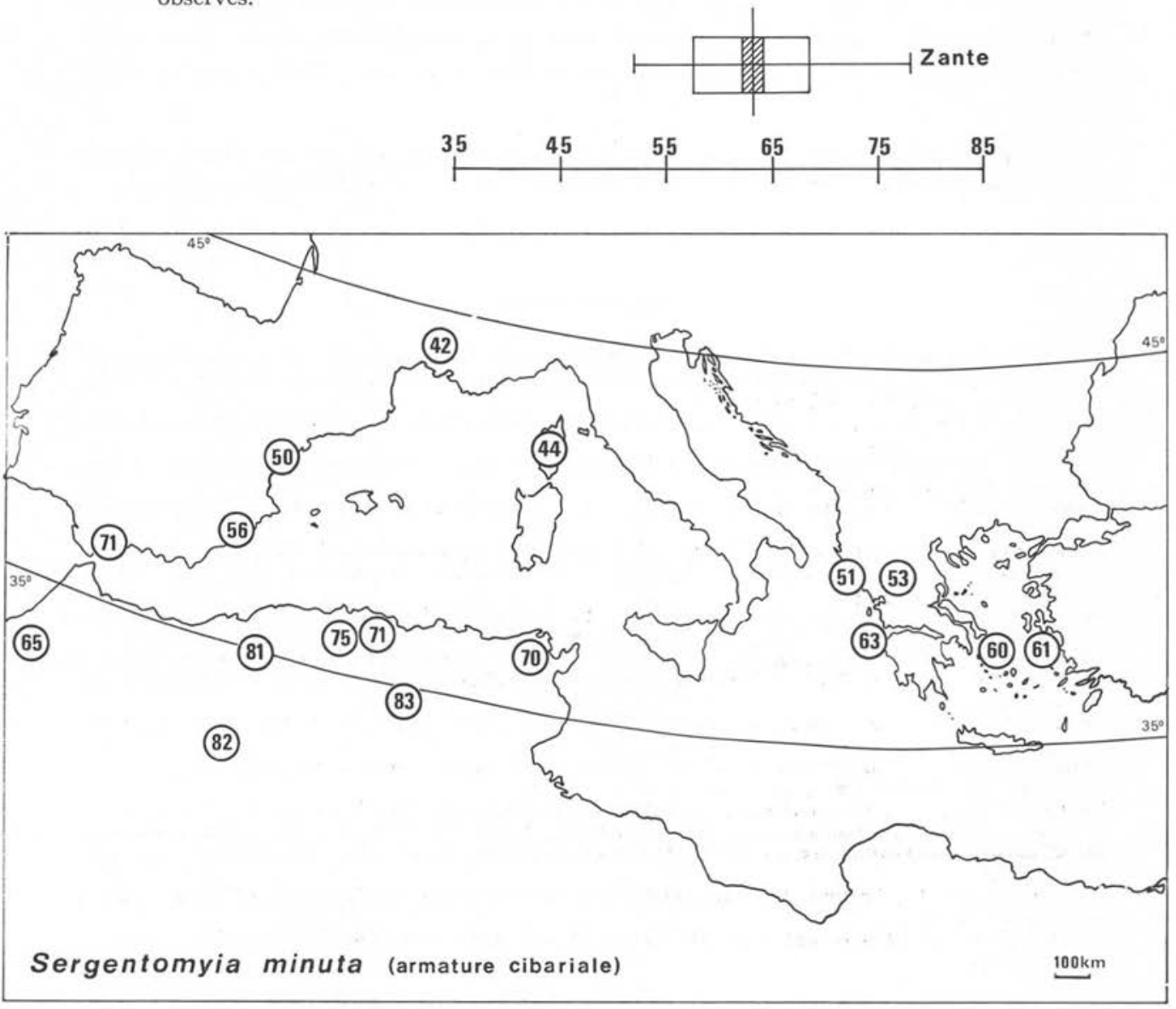

FIG. 8. - Valeurs moyennes du nombre de dents cibariales de $S$. minuta dans différentes régions du bassin méditerranéen. 


\section{3 - Conclusion}

La présence, en grand nombre, des deux vecteurs les plus probables de leishmaniose en Grèce : $P$. major à Corfou et Céphalonie et $P$. sergenti à Zante explique que le foyer des îles Ioniennes soit si actif. Le rôle accessoire joué par d'autres espèces comme $P$. perfiliewi et $P$. tobbi pourrait ne pas être négligeable.

La comparaison de ces îles avec celles de la mer Égée, qui s'opposent tant par leur caractères géographiques (localisation, géologie et biogéographie) que par leur situation épidémiologique, a permis de mettre en évidence un certain nombre de différences dans les peuplements de phlébotomes :

- absence quasi totale de $P$. perfiliewi à l'est, permettant de situer la limite orientale de son aire de répartition à cette latitude - prépondérance de $P$. simici en mer Égée - absence de $S$. dentata dans les Cyclades.

Il s'agit là de différences minimes, contrairement à ce que nous attendions en commençant cette étude. Bien que les cas de leishmaniose humaine répertoriés soient peu nombreux dans les îles de l'est, les vecteurs sont omniprésents, et elles constituent des foyers potentiels dans la perspective de modifications des peuplements humains.

Cette étude a bénéficié d'un appui financier du Programme spécial PNUD/Banque mondiale/OMS pour la recherche et la formation concernant les maladies tropicales.

\section{BIBLIOGRAPHIE}

AdLer S., Theodor O. : Investigations on Mediterranean kala azar. III - The sandflies of the Mediterranean basin. Distribution and bionomics of sandflies in Catania and district. Proc. R. Soc., I931, (B), $108,464-480$.

Adler S., Theodor O., Lourie E. M. : On sandflies from Persia and Palestine. Bull. Ent. Res., I930, $21,529-539$.

Adler S., Theodor O., Witenberg G. : A study of leishmaniasis in Canea, Crete. Proc. R. Soc., I938, (B), I $25,49 \mathrm{I}-5 \mathrm{I} 6$.

Annandale N. : The Indian Species of Papataci fly (Phlebotomus). Rec. Indian. Mus., I910, 4 35-52.

Artavanis S., Tsoutsanis K., Vrakas A., Tsilios C. : Epizootiological investigation of leishmaniasis (Leishmania donovani) in dogs on the island of Cephallonia. Delt. Hel. Ktenia. Het., 1982, 33, 31-38.

Artemiev M. M. : Révision des phlébotomes du sous-genre Adlerius (Diptera, Phlebotominae, Phlebotomus). Zool. Zh., I980, s9, I I 77-II92 (en russe).

Belazzoug S., Mahzoul D., AdDAdi K., Dedet J. P. : Sergentomyia minuta parroti (Adler et Theodor, 1927) en Algérie (Diptera, Psychodidae). Ann. Parasitol. Hum. Comp., 1982, 57, 621-630.

Caminopetros J. : Sur la faune des phlébotomes de la Grèce. Bull. Soc. Pathol. Exot., I934, 27, 450-455.

Caminopetros J. : Addition à la liste des phlébotomes signalés pour la première fois en Grèce. Bull. Soc. Pathol. Exot., 1935, 28, 44-46.

Croset H., Rioux J. A., Leger N., Houin R., Cadi Soussi M., Ben Mansour N., Maistre M. : Les méthodes d'échantillonnage des populations de phlébotomes en région méditerranéenne. Colloques internationaux du C.N.R.S. Écologie des leishmanioses, Montpellier, 1977, 239, I39-I5I.

Hadjinicolaou J. : Present status of Phlebotomus in certain areas of Greece. Bull. O.M.S., I958, 19. $967-979$.

Hertig M. : Phlebotomus and residual DDT in Greece and Italy. Am. J. Trop.Med., I949, 29, 773-809. 
Hourn R., Abonnenc E., Deniau M. : Phlébotomes du sud de la Turquie. Ann. Parasitol. Hum. Comp., 1971, 46, 633-652.

Leger N., Saratsiotis A., Pesson B., Leger P. : La leishmaniose en Grèce. Résultats d'une enquête entomologique effectuée en juin r977. Ann. Parasitol. Hum. Comp., I979, s4, I I-29.

Lewis D. J. : A taxonomic review of the genus Phlebotomus (Diptera, Psychodidae). Bull. Br. Mus. Nat. Hist. (Ent)., I982, 45, I $21-209$.

Madulo-Leblond G. : Les phlébotomes des Iles Ioniennes. Thèse Doc. Pharm. Reims, I983.

Newstead R. : On the genus Phlebotomus. Part III. Bull. Ent. Res., 1916, 7, I9I-192.

Nitzulescu V. : Sur la présence en Europe du Phlebotomus major s. str. Ann. Parasitol. Hum. Comp., 1930, 8, 376-381.

Nrtzulescu V.: A propos du Phlebotomus chinensis. Ann. Parasitol. Hum. Comp., I931, 9, 26r265.

PARrot L. : Sur un nouveau phlébotome algérien : Phlebotomus sergenti sp. nov. Bull. Soc. Pathol. Exot., 1917, I0, 564-567.

PARROT L. : Notes sur les phlébotomes II. Sur quelques phlébotomes de la Bokhara (URSS). Arch. Inst. Pasteur Algér., 1928, 6, 26-34.

Parrot L. : Notes sur les phlébotomes : Phlebotomus perfiliewi n. sp. Arch. Inst. Pasteur Algér., I930, $8,383-385$.

PARrot L. : Notes sur les phlébotomes XIV Phlébotomes de Grèce. Arch. Inst. Pasteur Algér., $1935,13,249-256$.

Perfiliew P. P. : Phlébotomes. Faune de l'URSS, Acad. Sci. Moscou Ed., 1937, ro, I-I44 (en russe).

Perfiliew P. P. : Phlebotominae. In Fauna of URSS : Diptera vol. III. Traduction anglaise : Israel program for scientific translations, Jerusalem, I968, 363 p.

Rioux J. A., Croset H., Leger N., Maistre M. : Remarques sur la taxonomie infraspécifique de Sergentomyia minuta, Sergentomyia africana et Sergentomyia antennata. Ann. Parasitol. Hum. Comp., 1975, so, 635-641.

RoNDANI C. : Species italicae generis Hebotomi Rndn, ex insectis dipteris : fragmentum septimum ad inserviendam dipteroligian italicam. Ann. Soc. Ent. Fr., 1843, 1, 263-267.

Scopoli J. A. : Deliciae faunae et florae insubricae, Pavie, I 786,85 p.

Sinton J. A. : The synonymy of the Asiatic species of Phlebotomus. Indian. J. Med. Res., 1928, $16,297-324$.

Sinton J. A. : Notes on some indian species of the genus Phlebotomus XXXII Phlebotomus dentatus $n$. sp., 1933, 20, 869-872.

Simıc C., Zrvkovic V. : La faune des phlébotomes de Yougoslavie et leur rôle dans l'épidémiologie de la fièvre à papatasi, du kala-azar et du bouton d'Orient. Arch. Inst. Pasteur Algér., I956, $34,380-387$.

Stephanides Th. : Corfu Phlebotomus (Dipt., Psychod.) found in human habitations. Bull. Ent. Res., 1939, 30, 303-304.

Stratigos J., Tosca A., Nicolis G., Papavasiliou S., Capetanakis J. : Epidemiology of cutaneous leishmaniasis in Greece. Inter. J. Dermatol., I980, 19, 86-88.

Theodor O. : Psychodidae - Phlebotominae. In : Die Fliegen der Palaearktischen Region. Schweizerbart, Stuttgart, $1958,55 \mathrm{p}$.

Theodor O. : Leishamaniasis. In : Van der Hoeden, Zoonoses. Elsevier, Amsterdam, 1964, 475-493.

Theodor O., Mesghali A. : On the Phlebotominae of Iran. J. Med. Ent., 1964, 13, 285-30o.

Tonnorr A. L. : Une nouvelle espèce européenne du genre Phlebotomus (Ph. neglectus). Ann. Soc. Ent. Belg., 1921, 61, 333-336.

Ward R. D., Bettini S., Maroli M., Mac Garry J. W., Draper A. : Phosphoglucomutase polymorphism in Phlebotomus perfiliewi perfiliewi Parrot, I930 (Diptera, Psychodidae) from central and northern Italy. Ann. Trop. Med. Parasitol., r981, 75, 653-66r. 